\title{
Gas Prediction of Sino-Singapore Tianjin Eco-City Based on ARMA
}

\author{
Model \\ Yizhi $\mathrm{Xu}^{1,2, \mathrm{a}}$, Peng Ling ${ }^{1, \mathrm{~b},{ }^{*}}$, Xiang $\mathrm{Li}^{1,2}$, Gaosheng $\mathrm{Li}^{1,2}$, Shaolong Cui ${ }^{1}$ \\ ${ }^{1}$ Institute of Remote Sensing and Digital Earth, CAS, Beijing 100094, China \\ ${ }^{2}$ University of Chinese Academy of Sciences, Beijing 100049, China \\ a762362967@qq.com, bpengling@radi.ac.cn
}

Keywords: time series analysis, ARMA, data aggregation platform, city pulsating

Abstract. With the development of Chinese economic growth, energy consumption is growing. Among them, the gas is an important energy source of urban living, and its consumption has increased year by year. In this paper, based on the data aggregation platform, and residents daily natural gas consumption data in SSTEC(Sino-Singapore Tianjin Eco-City)[1], considering the time series correlation of data, a time series prediction model ARMA was used to predict the daily consumption of gas in SSTEC.

\section{Introduction}

Energy is an important material basis of economic development. With the rapid economic growth and city expansion, China's energy problem is becoming more and more serious, showing a typical characteristics of high consumption and high waste. At present, China is still at its preliminary stage of industrialization and industrial restructuring, with rapid economic development and huge energy consumption ${ }^{[2]}$. And in the current structure of China's energy consumption, the fossil energy dominated ${ }^{[3]}$. Urban development means not only the expansion of the production sector, but also the strengthening and development of the city's comprehensive service functions of the tertiary industry growth. Urban population increment, improving living conditions, have occupied a major share in energy consumption ${ }^{[4]}$. After living energy structure adjustment and transformation, the energy consumption of urban residents has transformed from coal to gas.

Currently, energy consumption forecast mostly explores energy consumption influence factors and the law between the influence factors, such as the impact of urban population size, urban industrial structure as well as the number of industrial cities and so on, to explore their influence on urban energy consumption, and then build the appropriate model for prediction. In this paper, based on mathematical statistics theory, we adopt the autocorrelation moving average model (ARMA) to analysis the historical gas data, and conduct short-term prediction on the data. By exploring the objective laws of living daily gas consumption, we provided technical and information support for residential gas conservation, rational management of energy configuration, in order to achieve a reasonable energy planning and optimize energy consumption structure.

\section{Energy Pulsation Demand of SSTEC}

Along with the new-type urbanization become a national strategy object, the smart city construction is in full swing all over the country. The Ministry of Housing and Urban-Rural Development has been conducting smart city pilot project for three consecutive years, proposed a strict requirement for building geographic information public platform. Smart city public information platform is 
collecting more and more city data. Make full use of these data, analysis pulsating variation of urban development and relationship, has become an urgent need for Smart City construction.

SSTEC is the first batch of state-level Smart City pilot, one of the government strategic cooperation project between the China and Singapore. It has a profound understanding of smart city construction and rich experience in construction of smart city public information platform, which has formed some preliminary results and practical application. SSTEC's next target is to deepen and optimize infrastructure construction of public information platform, strengthen the data analysis and information mining, and make the platform become the core infrastructure of eco-city informationization construction and the exhibition of city development.

Based on the existing natural gas SSTEC historical daily consumption data, we used the ARMA model to make a short-term forecast for the residential daily gas consumption, which provides a reasonable suggestions for energy configuration.

\section{Research Methods}

Because of the periodic system of the time series in general geography of the Earth's revolution, and the moon orbiting solar activity, and therefore many phenomena have a certain seasonality such as the water level of rivers and the growth of the organism. Related to this, many human geography phenomenon due to seasonal changes in the ecological environment is also showing definite cycle regularity. Time series analysis is one of the quantitative forecasting methods and its basic principles: First, recognize the continuity of the development of things. Application of past data, we can speculate that the development trend of things. Second, considering the randomness of the development of things. Anything development are likely to suffer accidental factors. Time series forecasting in general reflect actual changes in three kinds of rules: trends, cyclical changes, random changes. For prediction, the approximation of a complex reality is more important rather than the model is $100 \%$ precisely. Whether a time series model building and forecasting success or not depends on the simple and accurately approximation. Currently there are three approximate expression: one is Moving Average, (MA), the other is Auto-Regression, (AR), the third is Auto-Regressive Moving Average, (ARMA). ARMA model which shows that both time series data sequences associated with the delay, but also about the error delay sequence, used to identify the seasonal time series data. Due to the daily consumption of natural gas and seasonal residents of a definite link, so this paper ARMA model is for time series analysis.

Residential gas consumption has a seasonal, cyclical properties. The consumption of natural gas daily adjacent to the number of days is relatively stable and a sharp increase or decrease in the substantial is absence, which can be regarded as stationary time series data, compliance with the precondition that time-series data analysis requires data periodically and smooth.

\section{ARMA Model Predictions}

Based on residential gas consumption data in SSTEC and time series forecasting, the forecasting process shows in figure 1. 


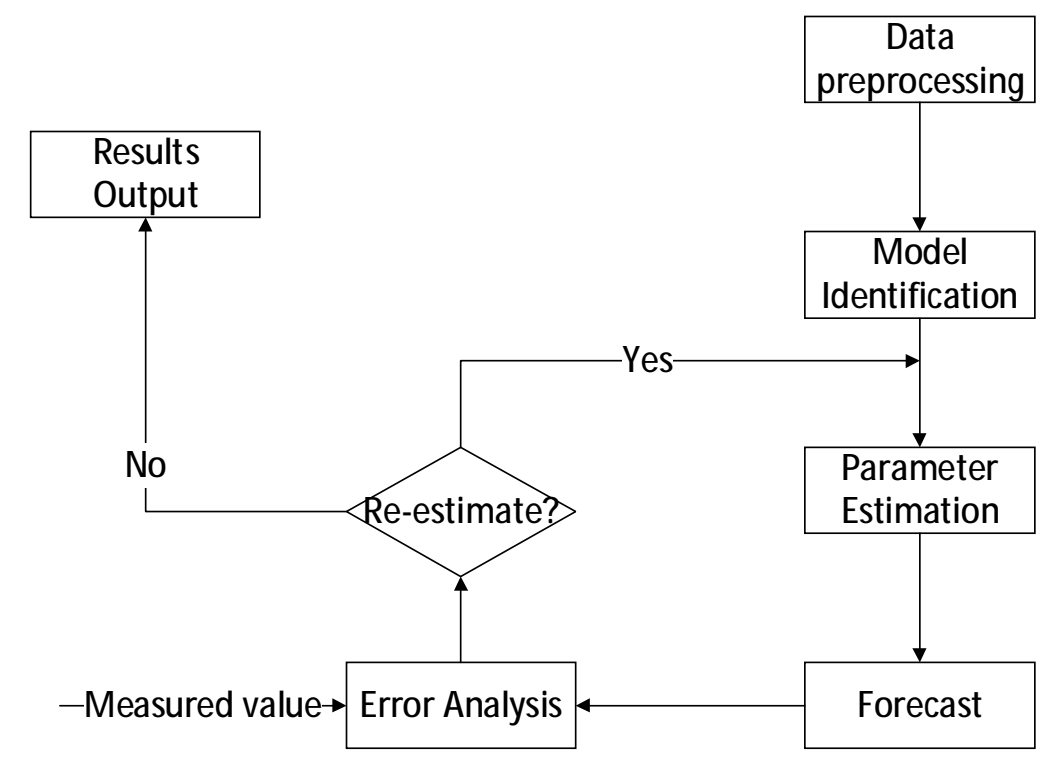

Fig. 1. The forecasting process of ARMA time series analysis

\section{ARMA time series analysis and forecasting process:}

1) Data Preprocessing: pre-processing of data includes zero mean processing and difference stationary process. Zero-mean process: the conversion from the original sample sequence mean which is non-zero mean sequence to zero mean sequence. Through data preprocessing, the original sequence satisfies the smooth and reversible precondition;

2) Model Identification: Using temporal autocorrelation function and partial autocorrelation function of time and space to determine the general direction of the model, to select the appropriate model as a candidate model and to determine the order of the model;

3) Parameter Estimation: by solving the equation to determine the parameter value;

4) Model Diagnosis: whether the original sequence and the error sequence of the model meets the random error test or not makes model diagnosis. Check in the approximate confidence interval parameter estimation of statistical significance, if the test is not passed, return to the step (2);

5) Forecast: Establish the model to predict the data;

6) Error Analysis: analyzed the error between the real value of data and predicted value. If the error exceeds the permitted range, to jump to the step (3) to re-estimate the parameters until error is in the permitted range; if error is in the permitted range, accept the model and model parameters and use to make prediction.

\section{Experiment and Analysis}

\section{Data presentation}

Thera is the data of living water consumption, gas consumption and heat consumption of Eco-city residents in the following table:

\begin{tabular}{|l|l|}
\hline Community & $\begin{array}{l}\text { Hong Shuwan, He Changyuan(1B), Mei Linyuan and other } \\
15 \text { district }\end{array}$ \\
\hline $\begin{array}{l}\text { The total number } \\
\text { of households }\end{array}$ & 22159 \\
\hline Monitoring period & From 1.1.2014 to 11.30.2014 \\
\hline Monitoring data & Living water, natural gas, thermal energy (heating) \\
\hline
\end{tabular}

In this paper, the SSTEC 2014 consumption data in each district of natural gas daily. 300 days were selected as the training data sample, and the following seven days of data were selected as the 
test data.

\section{Model Training}

Before using the ARMA model, we need to detect the stationary of the data sequence, we use $\mathrm{ADF}$ unit root test. Upon examination, the approximate sequence and details of the sequence of the test statistic P-value were less than 0.001, by ADF test, stationary series, ARMA model can be used for modeling. Model order using the AIC criterion to determine the minimum law. $\mathrm{p}$ is $5, \mathrm{q}$ is 3 and the model is the ARMA $(5,3)$ model.

ARMA $(5,3)$ model are established and the forecasting results of the time series analysis are shown in figure 2.

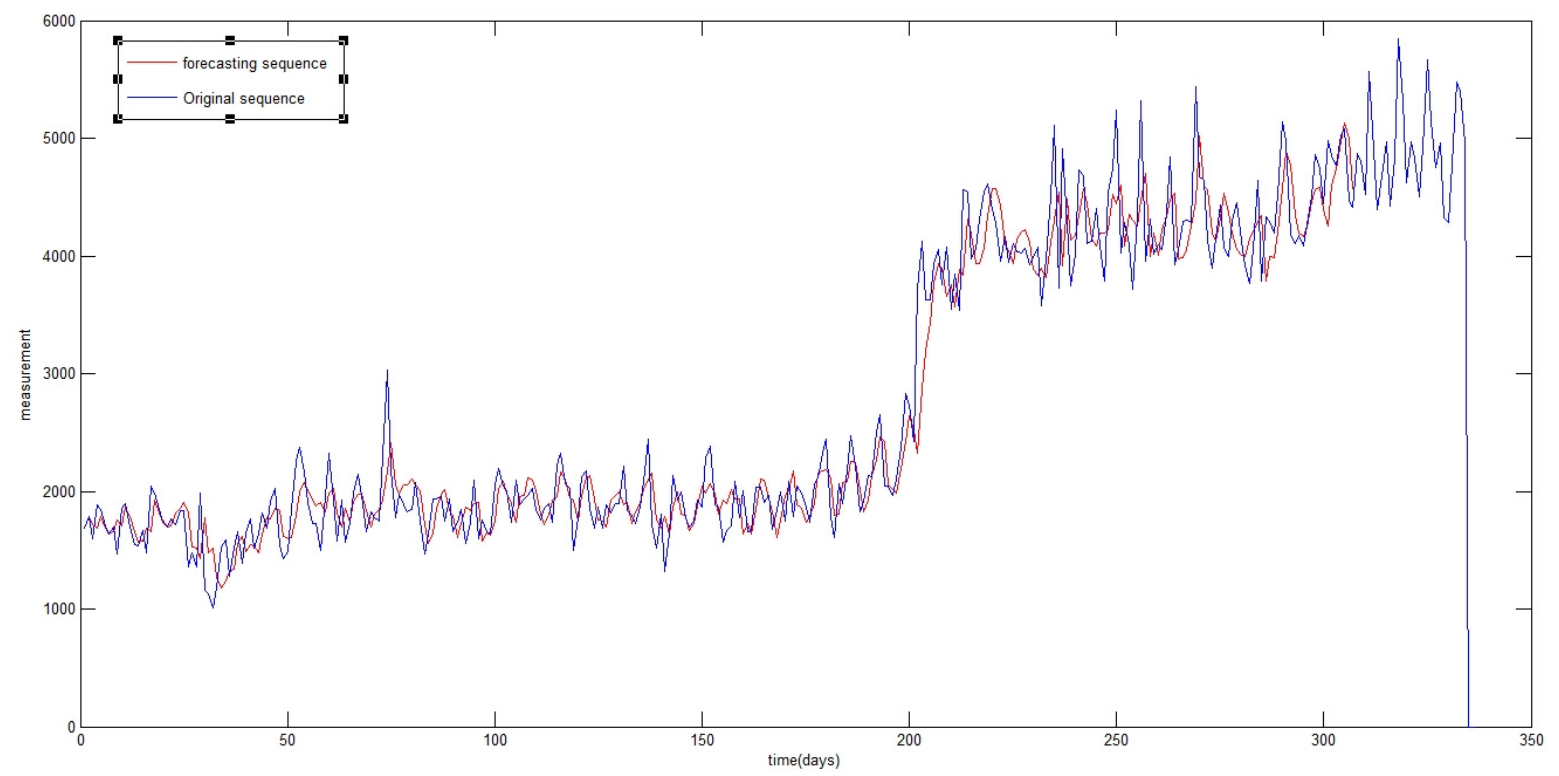

Fig.2. The comparison of the forecasting sequence and the original sequence

As can be seen from the figure forecasting sequence and the original sequence curve, they are similar, indicating that the forecasting of ARMA time series analysis has a good effect on daily consumption of natural gas in the Tianjin Eco-City.

Table 1 shows the prediction accuracy.

Table 1. Model fitting accuracy and model prediction accuracy

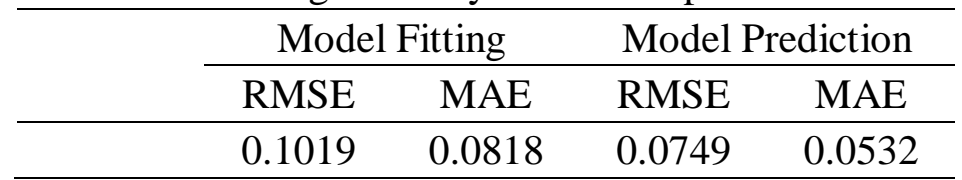

Table 2. Models predict the actual and predicted values

\begin{tabular}{cccc}
\hline Days & The Real Value & $\begin{array}{c}\text { The Predictive } \\
\text { value }\end{array}$ & $\begin{array}{c}\text { The Relative } \\
\text { Error }\end{array}$ \\
\hline 1 & 4985 & 4252.372 & 0.146966 \\
\hline 2 & 4848 & 4598.757 & 0.051412 \\
\hline 3 & 4767 & 4741.495 & 0.00535 \\
\hline 4 & 5025 & 4971.826 & 0.010582 \\
\hline 5 & 5075.4 & 5123.818 & 0.00954 \\
\hline 6 & 4469.4 & 4999.224 & 0.118545 \\
\hline 7 & 4415 & 4561.869 & 0.033266 \\
\hline
\end{tabular}

The results in Table 1 and Table 2 show that RMSE (relative root mean square error) and MAE (mean absolute error relative) of model training and model testing are small, which proves the model fit well and predictive value is close to the real value. 


\section{Experimental results}

Results analysis:

(1)As gas daily consumption which is related to residents living closely belong stationary series, ARMA model overall fitting error and the prediction error is small, high prediction accuracy.

(2) Through analysis on the results of every single forecast, ARMA model error is generally small, high prediction accuracy.

\section{Conclusion}

ARMA forecasting model are applied herein and the time series of gas daily consumption empirical analysis was used. Judging from the results, ARMA time series analysis model has good effect in forecasting residential natural gas daily consumption. That the energy consumption in spring was less than it in the fall and winter showed a gradual upward trend. The results reflect that the daily consumption of natural gas has a strong correlation with cyclical time for consumption of natural gas of residents in Tianjin Eco-city and it suggests that the ARMA model works very well in the gas consumption in Tianjin Eco-city. Besides, the analysis model at this stage considers only the time information. The spatial information and a comprehensive analysis of the impact and the space and time dimension of the neighboring region of its consumption of natural gas daily will be considered to be added to the model in the follow-up research, trying to combine both the time factor and the space factor of 15 districts in SSTEC, and further improve the new residential consumption of natural gas daily forecast model used in the Tianjin Eco-city, and gradually applied to the other energy data such as living water, heating, etc. and finally the SSTEC living energy data prediction system will form.

\section{Reference}

[1]. Yang Lina, Chi Tianhe, Peng Ling. Smart City Spatial Information Notice platform. 2014, BeiJing: Science Press.

[2]. Wu Qiaosheng, Cheng Jinhua. Analysis of China in the energy intensity of industrial change and Factors - An Empirical Analysis Based on Decomposition Model. Finance And Economics, 2006(06): 75-85.

[3]. Xu Shichun, Xi Rong, He Zhengxia. Factors Affecting China's energy consumption and carbon emissions analysis Policy Implications. Resources Science, 2012(01): 2-12.

[4]. Yao Yongling. Beijing urban development affect energy consumption factors. China Population, Resources and Environment, 2011(07): 40-45. 